\title{
Sustainable Biocomposites from Recycled Bale Wrap Plastic and Agave Fiber: Processing and Property Evaluation
}

\author{
Iftekhar H. Chowdhury, Mohamed A. Abdelwahab, Manjusri Misra,* and Amar K. Mohanty
}

Cite This: ACS Omega 2021, 6, 2856-2864

Read Online

ABSTRACT: Plastic recycling to make sustainable materials is considered one of the biggest initiatives toward a greener environment and socioeconomic development. This research aims to investigate the properties of a blend of recycled bale wrap linear low-density polyethylene (rLLDPE) and polypropylene (PP) (rLLDPE/PP 50:50 wt \% matrix), which was further reinforced with 25 wt \% agave fiber prepared by injection-molding. Different ratios of a combined industrial compatibilizer (maleic anhydridegrafted $\mathrm{PP} / \mathrm{PE}$ ) were used (1-3 wt \%), which were compared with a synthesized compatibilizer made from maleic anhydride-PP/ rLLDPE in terms of mechanical and thermomechanical properties of the biocomposites. Incorporation of the compatibilizer in the

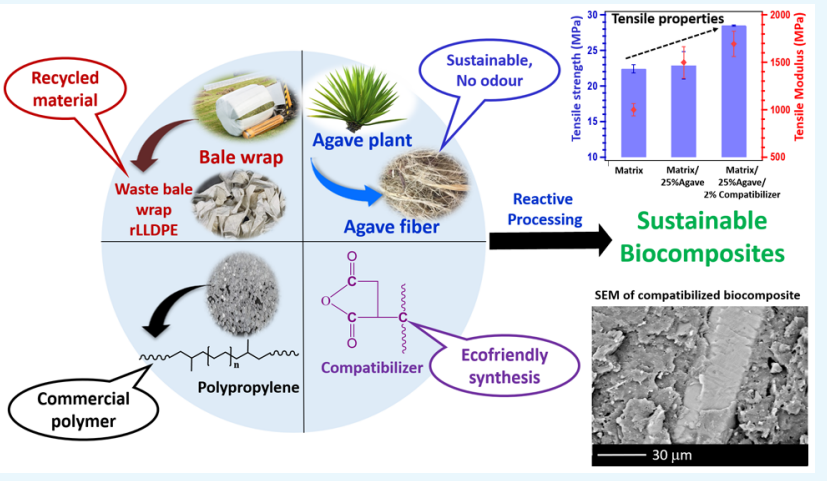
composite improved the interfacial adhesion between the hydrophobic matrix and the hydrophilic agave fiber, which further increased the mechanical properties and heat deflection temperature of the composite. Scanning electron microscopy showed enhanced compatibility and adhesion between the fiber and the matrix by inclusion of 2 wt \% compatibilizer. The synthesized compatibilizer-blended composite showed better mechanical properties than the industrial one, which indicates the potential application of this composite (around 62\% recycled material) in the manufacture of packaging materials and commodity products.

\section{INTRODUCTION}

Sustainable biocomposites are an emerging class of materials, promising an alternative to traditional plastics. The application of recycled polymers in composites is an urgent need to reduce the neat polymer content and find value-added applications for the waste plastic which is affecting the environment. It is estimated that 300 million tons of plastic are produced every year; $50 \%$ of the products are for single-use purposes. ${ }^{1}$ Most of these plastics are landfilled, incinerated, or dumped in the ocean. ${ }^{2}$ Therefore, plastic recycling has drawn significant interest in various industries.

Linear low-density polyethylene (LLDPE) is one of the essential commodity plastics that are being used for the production of various packaging films, containers, and other molded parts. ${ }^{3-5}$ LLDPE is well known for having excellent flexibility, impact strength, and durability but has low tensile and flexural strength. It has shorter branches, which is why its chains are able to move against one another without entangling together when elongated. LLDPE also has excellent recycling characteristics. It can be recycled multiple times as it maintains its base properties during the recycling process. ${ }^{6}$ In Ontario, over 3500 tons of plastic agricultural waste is generated each year, including 2721 tons from plastic bale and silage wraps that are essentially made of LLDPE. ${ }^{7}$ The last few decades have brought increased interest in the production of these bale wraps, which have been primarily used for the storage of forage. There are many advantages of bale wraps such as better leaf retention compared to dry hay, no storage structures needed, and reduction of weather risk. Furthermore, the bale wrap has great potential to be reused and recycled because of having high elongation, high impact strength, low capital cost, and so forth. Bale wrap LLDPE can be recycled and blended with another commercial polymer to produce a sustainable blend as an alternative to the nonrenewable commercial option.

Polypropylene (PP) is among the cheapest and most used plastics available today, having good tensile and flexural properties but relatively low impact strength. It has a linear hydrocarbon structure similar to LLDPE. PP materials have a wide variety of applications in the automotive, packaging, and construction industries. ${ }^{8}$ Researchers have investigated PP materials blended with various recycled thermoplastics such as polyester, high-density polyethylene (HDPE), and polyethylene (PE). ${ }^{9-11}$ No significant research was found regarding

Received: October 24, 2020

Accepted: December 21, 2020

Published: January 19, 2021 
Table 1. Formulations of the Biocomposites ${ }^{a}$

\begin{tabular}{lccccc}
\multicolumn{1}{c}{ code } & $\begin{array}{c}\text { agave fiber } \\
\text { (wt \%) }\end{array}$ & $\begin{array}{c}\text { PP (wt } \\
\%)\end{array}$ & $\begin{array}{c}\text { rLLDPE } \\
(\text { wt \%) }\end{array}$ & $\begin{array}{c}\text { Industrial compatibilizer (MAPP/MAPE) } \\
\text { ratio (50:50) (wt \%) }\end{array}$ & $\begin{array}{c}\text { Synthesized compatibilizer MA(PP/rLLDPE) } \\
\text { ratio (50:50) }\end{array}$ \\
PP & 0 & 100 & 0 & 0 & 0 \\
rLLDPE & 0 & 0 & 100 & 0 \\
PP/rLLDPE 50:50 & 0 & 50 & 50 & 0 & 0 \\
25\% agave & 25 & 37.5 & 37.5 & 1 & 0 \\
1\% ind comp & 25 & 37 & 37 & 2 & 0 \\
2\% ind comp & 25 & 36.5 & 36.5 & 3 & 0 \\
3\% ind comp & 25 & 36 & 36 & 0 & 0 \\
1\% syn comp & 25 & 37 & 37 & 0
\end{tabular}

${ }^{a}$ Ind comp: industrial compatibilizer; syn comp: synthesized compatibilizer.

blending PP with recycled LLDPE to produce packaging products having excellent tensile and flexural properties as well as good impact strength. Therefore, PP could be an excellent choice to be investigated as a blend with LLDPE.

Natural fiber-based composites are also considered one of the great sustainable alternatives to the synthetic material based composites. Natural fibers have various significant advantages over their synthetic counterparts such as abundant availability, lower cost, lower environmental impact, and easier processing. ${ }^{12,13}$ Among natural fibers, agave fiber has drawn significant attention for making automotive parts. Ford is considered one of the leading automobile manufacturing companies and has researched and utilized sustainable biobased materials in their vehicles since the year 2000. ${ }^{14,15}$ Agave fibers are derived from the Agave americana plant and it is largely produced as a coproduct in the tequila industry. ${ }^{15,16}$ It contains $68-80 \%$ cellulose, $15 \%$ hemicellulose, 5-7\% lignin, and $0.26 \%$ wax. $^{17,18}$ Despite agave fiber-based composites having many desirable properties, they have been the subject of little published research. Annandarajah et al. ${ }^{18}$ found that the highest elastic moduli and yield stress were reached at $20 \mathrm{wt} \%$ agave fiber blended separately with LLDPE, HDPE, and PP. Singha and Rana ${ }^{13}$ showed that polystyrene materials blended with 20 wt $\%$ agave fiber had the highest mechanical characteristics. Other researchers have used different natural fillers to obtain sustainable biocomposites. Youssef et al. ${ }^{19}$ prepared composites with corn husk fibers and recycled LDPE by melt extrusion and found increased tensile properties but decreased hardness with an increase in fiber loading. Lei et al. ${ }^{20}$ investigated recycled HDPE with bagasse fiber and described a similar tendency, an improvement in the moduli by about $50 \%$ by incorporation of $30 \mathrm{wt} \%$ of bagasse fiber. Hence, it is deduced that around $25 \mathrm{wt} \%$ of the natural filler in the composites gives optimum mechanical properties.

The mechanical characteristics of the natural filler-based thermoplastic composites can be improved by the incorporation of a suitable compatibilizer in the polymer matrix. Thermoplastic polymers such as $\mathrm{PP}$, polyethylene terephthalate (PET), polybutylene succinate (PBS), and so forth have been combined with natural fillers such as miscanthus, lignin, and wood to produce biobased sustainable composites that can be used in automotive, packaging, and electronics industries. ${ }^{21}$ Natural fillers have various advantages over conventional synthetic fillers, as described earlier, but exhibit poor mechanical properties when blended in the composites. These natural fillers are hydrophilic and thus are incompatible with the hydrophobic matrix polymer. ${ }^{22}$ A suitable compatibil- izer can help to overcome this shortcoming by enhancing adhesion between the fiber and matrix phases and consequently enhancing the mechanical properties of the composite. Abdelwahab et al. ${ }^{23}$ found that a combination of MAPP and EBGMA compatibilizer provided an improved tensile, flexural, and impact strength, which could not be achieved using only MAPP or EBGMA. Zhang et al. ${ }^{24}$ found that a single compatibilizer system such as MAPP only improves the flexural and tensile strength of the recycled PET/ PP blend but reduced its impact strength, while a mixture of MAPP and POE-MA or EVA-MA maintains the balance between all significant mechanical properties. Muthuraj et al. ${ }^{25}$ observed that compatibilized composites displayed better fiber-matrix interaction, whereas the uncompatibilized composites showed a poor interface between the fiber and matrix phases. It was reported that tensile, flexural, and impact strength increased by $37.5,18$, and $59 \%$, respectively, for composites containing 5 wt \% MA- $g$-PBS/PBAT compatibilizer and $30 \%$ miscanthus fiber compared to composites with $30 \%$ miscanthus fiber but without a compatibilizer. Gao et al. ${ }^{26}$ also reported that grafting modification by the MA compatibilizer enhanced the flexural and tensile properties of the $\mathrm{PP} / \mathrm{PE}$ composite materials having wood particles as a natural filler. Thus, the incorporation of a suitable compatibilizer is essential to enhance the mechanical properties of a thermoplastic composite by enhancing the compatibility of the phases in the composite system.

The aim of this research is to study the effect on the performance of adding recycled LLDPE (bale wrap) and agave fiber to neat PP with a compatibilizer content variation of $1-3$ wt \%. Incorporation of a synthesized compatibilizer (MAPP/ LLDPE) in the composite helps to improve the interfacial adhesion between the two different polymers (PP and recycled LLDPE) and natural filler (agave fiber), which will further increase the mechanical performance of the composite.

\section{RESULTS AND DISCUSSION}

2.1. Mechanical Characteristics. The biocomposites with 25 wt \% agave fiber were fabricated by adding different amounts $(1,2$, and $3 \mathrm{wt} \%)$ of a synthesized or industrial compatibilizer (Table 1). Figure 1a displays the comparison of the effect of synthesized and industrial compatibilizers on tensile and flexural moduli of the composites. The stressstrain curve of the matrix and the composites are shown in Figure S1. Neat PP had a tensile strength of $38.4 \mathrm{MPa}$ while having a lower impact strength $(22 \mathrm{~J} / \mathrm{m})$. On the other hand, recycled bale wrap linear low-density polyethylene (rLLDPE) 


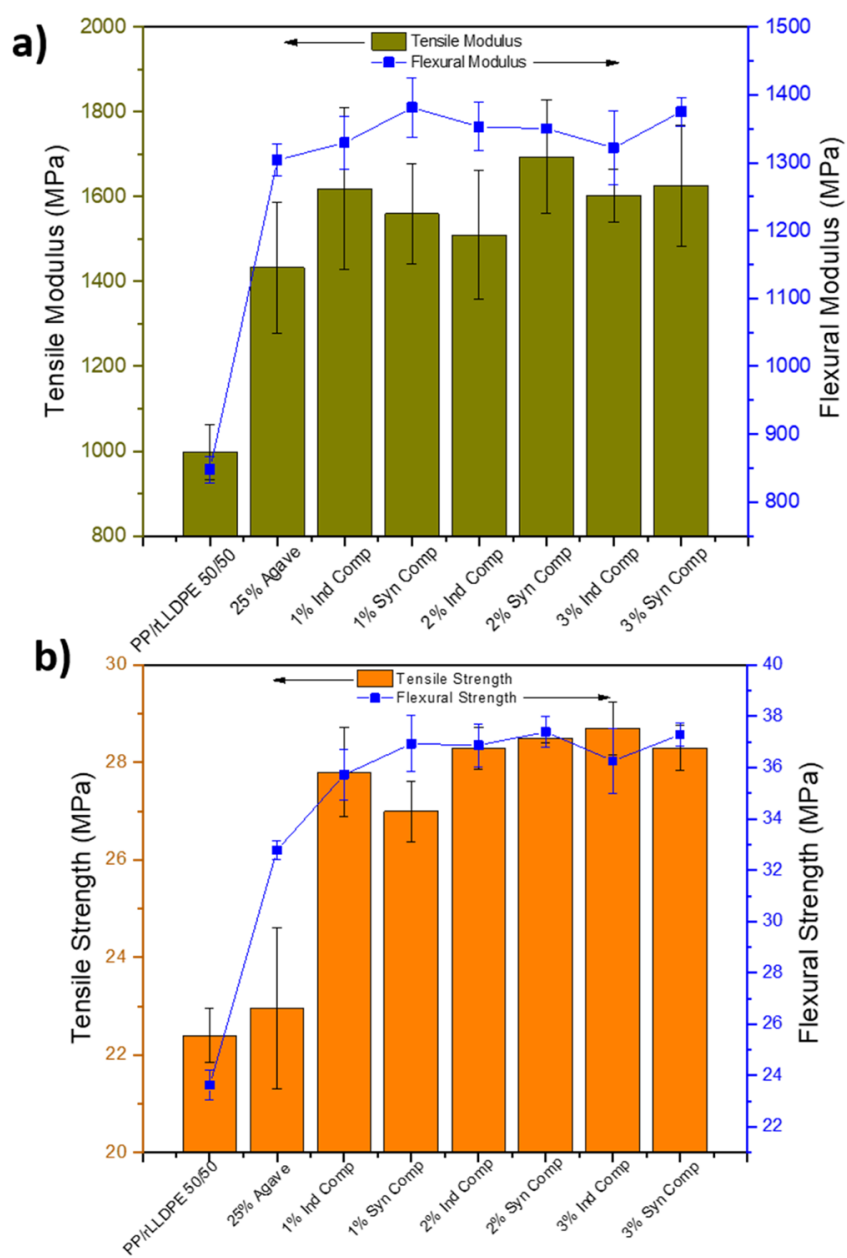

Figure 1. (a) Tensile and flexural moduli and (b) tensile and flexural strengths of the composite prepared with the synthesized compatibilizer (Syn Comp) and industrial compatibilizer (Ind Comp).

showed a lower tensile strength of $18.7 \mathrm{MPa}$, having a high impact strength of $435.98 \mathrm{~J} / \mathrm{m}$. Thus, to have an optimum property balance between strength and toughness, we used the matrix of PP and rLLDPE in a 50:50 ratio. The agave fiber acts as a filler which greatly enhances the tensile and flexural modulus of the biocomposite. It was reported that the addition of agave fiber increases the mechanical properties; the maximum elastic modulus was observed with a loading of 20 wt $\%$ agave fiber for agave/LLDPE and agave/PP composites and the maximum tensile strength was obtained at $30 \mathrm{wt} \%$ fiber loading. ${ }^{18}$ Hence, 25 wt \% fiber loading was selected for the composite, which also included the compatibilizer. The prepared agave/matrix showed a similar trend of enhancement of the tensile and flexural moduli by 50.1 and $53.8 \%$, respectively, with the incorporation of $25 \mathrm{wt} \%$ agave fiber (Figure 1). The enhancement of the modulus is consistent with previous work with the incorporation of high modulus agave fiber to the matrix..$^{27,28}$ Lei et al. ${ }^{20}$ investigated recycled HDPE with bagasse fiber and reported that a modulus increase of about $50 \%$ was obtained with the addition of $30 \mathrm{wt} \%$ bagasse fiber. The addition of agave fiber to the PP/rLLDPE matrix facilitates stress transformation from the matrix to the filler, which increased the modulus of the composite. The tensile and flexural strengths of the matrix and the composites are shown in Figure 1b. Similar results were obtained for the strength; incorporation of $25 \mathrm{wt} \%$ agave fiber greatly enhanced the tensile and flexural strength of the biocomposite. The flexural strength increased from 23.6 to $32.8 \mathrm{MPa}(38.9 \%$ increase) with the addition of 25 wt $\%$ fiber.

To increase the fiber-matrix interaction, the further addition of the MAPP/rLLDPE compatibilizer led to an improvement in both strength and modulus (Figure 1). This inclusion proves the positive adhesion effect of the compatibilizer, hence increasing the amount of stress transfer from the agave filler to the PP/rLLDPE matrix. In Figure 1, both synthesized and industrial compatibilizers showed improvement in both the tensile strength and modulus of the composite. The compatibilizer content was varied from 1 to $3 \mathrm{wt} \%$ of the composite and $2 \mathrm{wt} \%$ compatibilizer content showed the optimum result.

In the case of the synthesized compatibilizer, higher tensile and flexural moduli were achieved because of the better mixing of the compatibilizer and the filler in the composite. This is related to the synthesis procedure of these two types of polymer (PP and rLLDPE). In the case of synthesized compatibilizer production, the MA, PP, and rLLDPE were all mixed in the extruder; however, in the case of the industrial compatibilizer system, MAPP and MAPE were combined in the extruder with the other components. The addition of $2 \mathrm{wt}$ $\%$ synthesized compatibilizer increased the tensile modulus of the composite from nearly 1000 to $1700 \mathrm{MPa}$, while 2 wt \% industrial compatibilizer increased it to $1500 \mathrm{MPa}$. Flexural modulus was also increased by the addition of the compatibilizer, for instance, $1350 \mathrm{MPa}$ was achieved for a 2 wt $\%$ compatibilizer, where the flexural modulus of the matrix was $850 \mathrm{MPa}$ (Figure 1).

The increment in strength can also be explained by the adhesion effect of the compatibilizer, which was also observed in the composite modulus increase. Improvement of flexural strength was observed at 25 wt \% fiber loading with 2 wt \% compatibilizer content showing optimum properties. The rigid character of agave fiber caused an increase in tensile and flexural strength. Figure 2 shows that around $37.5 \%$ increment in flexural strength with respect to the matrix flexural strength was achieved with $25 \%$ fiber loading, which could be further increased to $56.3 \%$ with the addition of 2 wt \% compatibilizer.

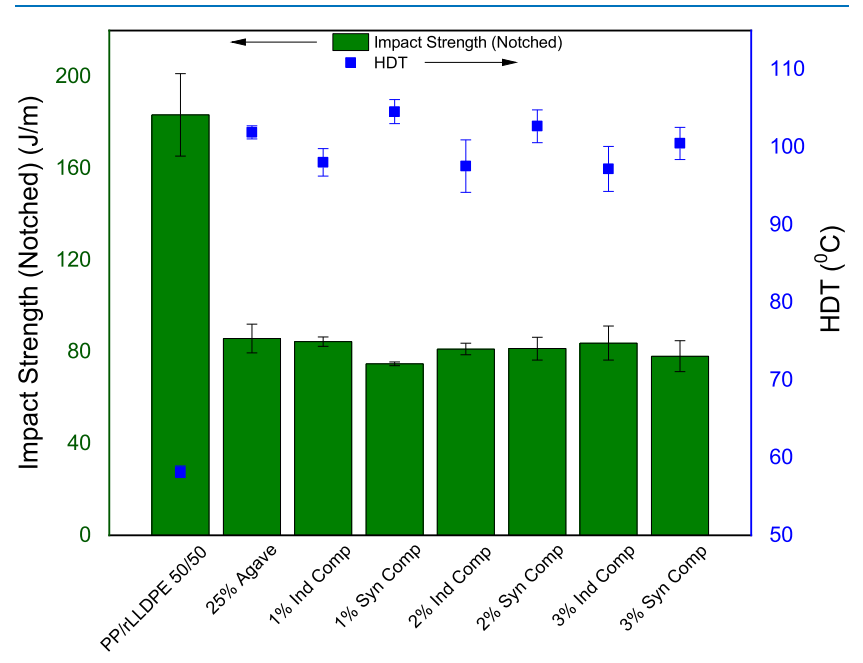

Figure 2. Izod Impact strength (notched) and HDT of the composites prepared with the synthesized compatibilizer (syn comp) and industrial compatibilizer (ind comp). 

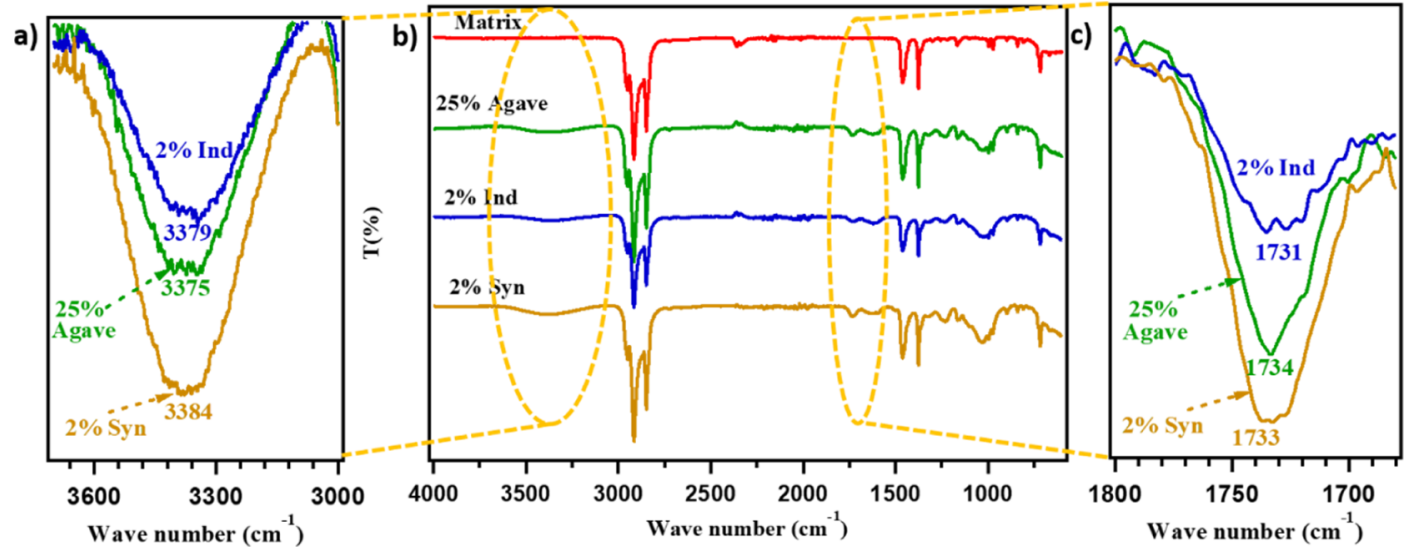

Figure 3. FTIR data of the matrix (PP/rLLDPE 50:50), $25 \mathrm{wt} \%$ Agave, $2 \%$ industrial comp (Ind), and $2 \%$ synthesized compatibilizer (Syn) from (a) $3000-3800 \mathrm{~cm}^{-1}$, (b) from $500-4000 \mathrm{~cm}^{-1}$, and (c) $1600-1800 \mathrm{~cm}^{-1}$.

Scheme 1. Possible Mechanism of the Reaction between Agave Fiber and the Bale Wrap rLLDPE and PP Matrix Prepared with an Industrial Compatibilizer (ind comp) or a Synthesized Compatibilizer (syn comp)

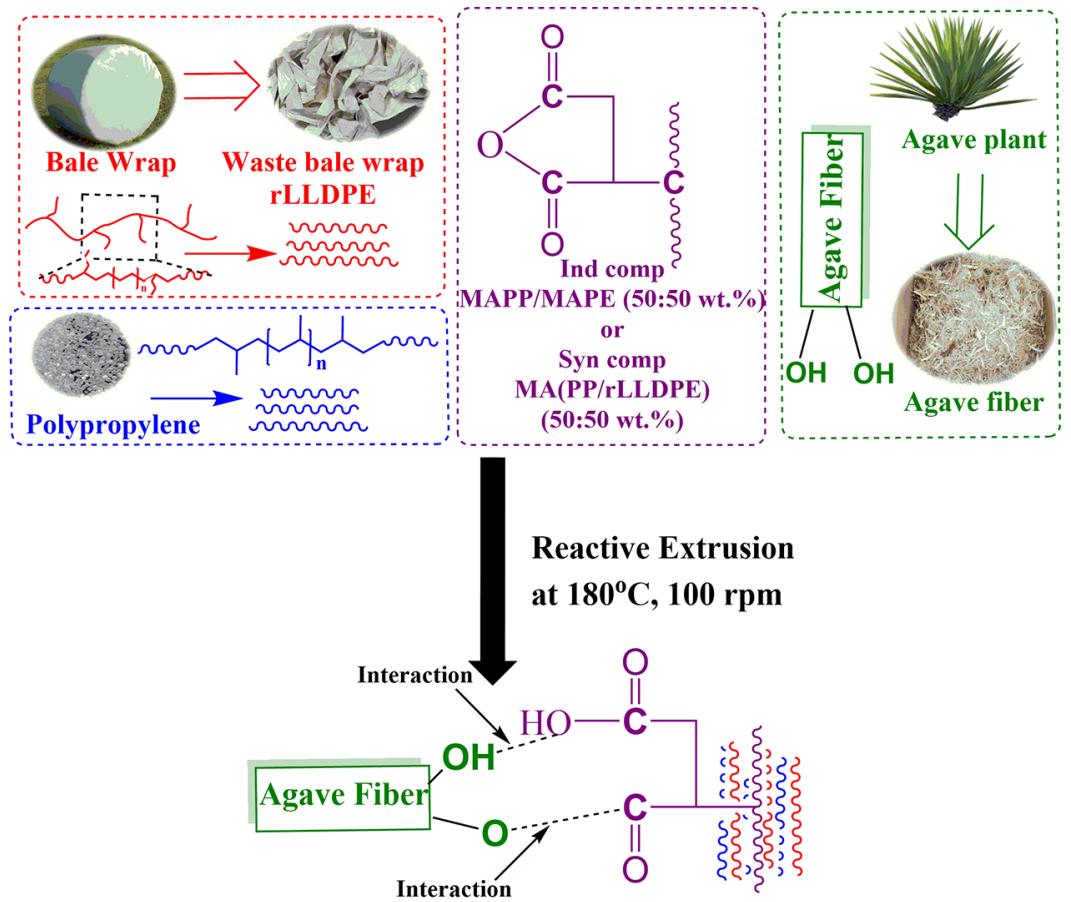

Sustainable Biocomposites

The notched Izod impact strength and heat deflection temperature (HDT) of agave/PP/rLLDPE composites are shown in Figure 2. Impact strength of the PP/rLLDPE matrix was found to be $183 \mathrm{~J} / \mathrm{m}$, which sharply decreased to $85 \mathrm{~J} / \mathrm{m}$ after the addition of 25 wt \% fiber loading. Previous research on agave fiber blended with PP or LLDPE also claimed that incorporation of the fiber reduced the impact strength. ${ }^{18,27,28}$ This is due to the hydrophilic nature of the agave fiber and the hydrophobic nature of the polyolefin (PP/rLLDPE) matrix, which gave rise to poor fiber-matrix interaction. The further addition of a MA-grafted compatibilizer to the system did not show any change in the impact strength of the composite compared to the uncompatibilized composite. Similar types of observations have been reported in earlier studies. ${ }^{22,29}$ Overall, the optimum sustainable composite was achieved using 2 wt \% of the synthesized compatibilizer; the tensile and flexural strengths increased by 24.4 and $14.1 \%$, respectively. Moreover, the tensile and flexural moduli increased by 13.1 and $3.5 \%$ compared to the composite without the compatibilizer.

2.2. Heat Deflection Temperature. The HDT is a significant physical property, which indicates the polymer's ability to retain its stiffness under specific temperature and load. This temperature also governs the design and application of the final material. Figure 2 displays the HDT of the PP/ rLLDPE matrix, which was found to be $58.2{ }^{\circ} \mathrm{C}$. The HDT value of the matrix reached $102{ }^{\circ} \mathrm{C}$ with the addition of $25 \mathrm{wt}$ $\%$ agave fiber, which was a nearly $75 \%$ increase. The improvement of the HDT values with the addition of agave fiber is due to the reinforcement effect of the filler and agrees with the enhancement of the modulus that was reported earlier in Figure 1. In general, the addition of natural fiber enhances the HDT of the composites. ${ }^{15,30}$ The addition of industrial 
compatibilizer (MAPP/MAPE) slightly lowered the HDT. For example, the HDT reduced from 102 to $98{ }^{\circ} \mathrm{C}$ with the inclusion of $1 \mathrm{wt} \%$ industrial compatibilizer to the composite sample. However, the addition of the synthesized compatibilizer enhanced the HDT of the composite. The addition of $1 \mathrm{wt}$ $\%$ synthesized compatibilizer enhanced the HDT by around 3 ${ }^{\circ} \mathrm{C}\left(105{ }^{\circ} \mathrm{C}\right)$ compared to an uncompatibilized composite. This indicates the resistance to the flow of the material at a high temperature and good interaction and a stable network between the matrix and agave fiber by the inclusion of the synthesized compatibilizer. Overall, the optimal sustainable composite of 25 wt \% agave fiber and 2 wt \% synthesized compatibilizer had a high tensile strength and modulus as well as HDT compared to the industrial compatibilizer and the uncompatibilized composite.

2.3. FTIR Characterization. FTIR spectra were used to estimate the interaction between the matrix (PP/rLLDPE 50:50) and agave fiber, with and without the compatibilizer, as shown in Figure 3. The expanded spectra in the region of $3000-3800$ and $1600-1800 \mathrm{~cm}^{-1}$ are shown in Figure $3 \mathrm{a}, \mathrm{c}$ for better clarification. The matrix displayed prominent peaks in the region of $1400-1460$ and $2850-2960 \mathrm{~cm}^{-1}$ corresponding to the $-\mathrm{CH}_{2}$ and $-\mathrm{CH}_{3}$ groups of $\mathrm{PP}$ and $\mathrm{PE}$. Incorporation of 25 wt \% agave fiber produced peaks at 1734 and $3375 \mathrm{~cm}^{-1}$ corresponding to the $\mathrm{C}=\mathrm{O}$ and $-\mathrm{OH}$ groups of the agave fiber. With the incorporation of the compatibilizer, the $\mathrm{C}=\mathrm{O}$ group broadened and increased in intensity, indicating the formation of an ester peak between the MA and the hydroxyl group of the agave fiber (Figure 3c). The broadness of the peak was more prominent in the case of the synthesized compatibilizer than for the industrial compatibilizer. Similar results of the interaction between MA and natural fiber have been observed in other studies. ${ }^{23}$ The difference in the intensity of the carbonyl group of the fiber with the incorporation of the compatibilizer confirms the interaction between the matrix and agave fiber, as shown in Scheme 1.

2.4. Morphological Characterization. Morphological characterizations of cryo-fractured surfaces of the PP/rLLDPE matrix and agave fiber composite samples, with and without a compatibilizer, were analyzed using SEM. A PP/rLLDPE 50:50 matrix showed a co-continuous morphology (Figure 4a) and it
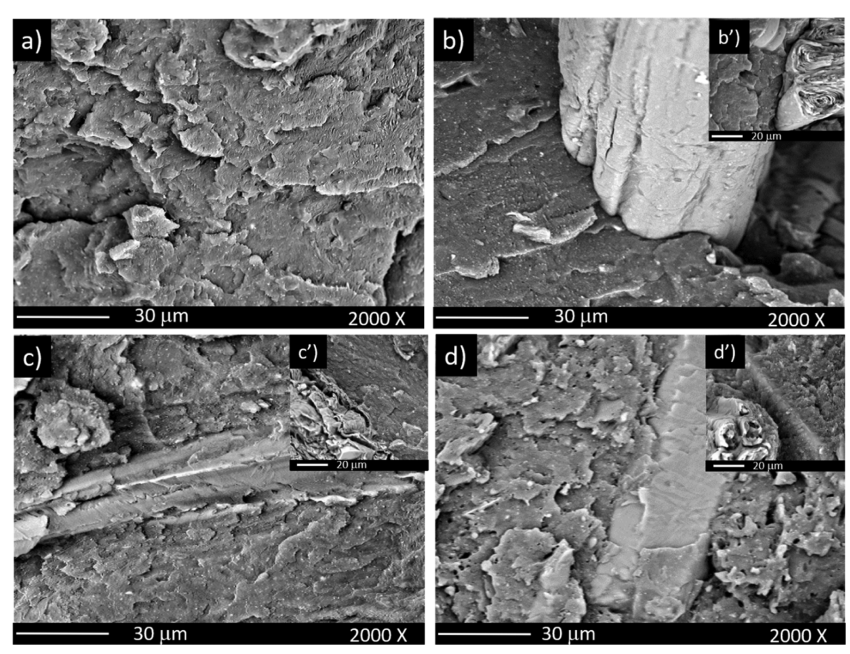

Figure 4. SEM images of cryofracture of (a) PP/rLLDPE 50:50; $\left(b, b^{\prime}\right) 25 \%$ agave; (c, $\left.c^{\prime}\right) 2 \%$ industrial compatibilizer (ind comp), and $\left(\mathrm{d}, \mathrm{d}^{\prime}\right) 2 \%$ synthesized compatibilizer (syn comp) samples. was very difficult to distinguish between the two polymers. Zhang and Ajji ${ }^{31}$ found that no cross-orientation was detected in the PP/LLDPE blend. Figure $4 b, b^{\prime}$ shows the phase separation and pull-out between the agave fibers and the matrix, indicating a weak interface and poor interaction between the matrix and the fiber. The inclusion of $2 \mathrm{wt} \%$ compatibilizer enhanced the compatibility and adhesion between the matrix and the fiber, as shown in Figure 4c,d. Cisneros-Lopez et al. ${ }^{32}$ obtained a good interface by blending the LLDPE/agave fiber in the presence of MAPE as a compatibilizer. However, there was not much difference between the use of industrial and synthesized compatibilizers, as shown in Figure 4. This result indicates that the compatibilizer played a role in the enhancement of the compatibility between the matrix and the agave fiber.

2.5. Rheological Characterization. Rheological properties are another important characteristic as they represent the flow of the polymer in its molten state. The complex viscosity and storage modulus of the matrix and biocomposites, with and without the compatibilizer, are shown in Figure 5. The neat PP/rLLDPE 50:50 matrix showed a reduction in the Newtonian plateau as compared to neat PP that was seen in a previous paper. ${ }^{33}$ At a higher frequency, the polymer matrix showed shear thinning behavior (Figure 5a). The complex viscosity and storage modulus of the PP/rLLDPE matrix increased after the inclusion of 25 wt \% agave fiber at lower and higher angular frequencies (Figure 5). Previous studies showed the same behavior that the complex viscosity and storage modulus increased with the incorporation of the natural fiber. ${ }^{34,35}$ This is due to the fiber hindering the mobility of the polymer chains and increasing collisions between the fiber particles. This behavior is more predominant at a lower frequency level. Incorporation of the agave fiber also decreased the Newtonian plateau, which disappears with the inclusion of the compatibilizer. This is due to the rigidity of the agave fiber in the polymer matrix, which restricts the movement of the matrix chains. ${ }^{23}$ At lower frequencies, the storage and loss modulus increased with the incorporation of the compatibilizer, resulting from the deformability of the dispersed phase of fiber particles in the matrix (Figure $5 b, c) .{ }^{17}$ Incorporation of the MA-grafted compatibilizer (synthesized or industrial compatibilizer) increased the complex viscosity at lower and higher frequencies because of improved compatibility and interfacial adhesion between the agave fiber and the matrix, as confirmed by SEM data. However, there was not much difference in rheological characteristics by using industrial versus synthesized compatibilizers, as shown in Figure 5.

2.6. Thermal Properties (DSC). Figure 6 and Table 2 show the DSC (second heating) of the PP/rLLDPE matrix and its composites. As the composite contains two types of polymer in the matrix, that is, PP and rLLDPE, there are two individual peaks. PP and rLLDPE in the matrix blend had melting temperatures $\left(T_{\mathrm{m}}\right)$ of 64 and $124{ }^{\circ} \mathrm{C}$, respectively. The $T_{\mathrm{m}}$ of rLLDPE and PP remained consistently unaffected in all composites at $\sim 124$ and $\sim 164{ }^{\circ} \mathrm{C}$, respectively. Annandarajah et al. ${ }^{18}$ showed no change in the $T_{\mathrm{m}}$ of polyolefin (PP, LLDPE, HDPE) by the inclusion of the agave fiber. The percentage crystallinity for all the samples also varied with compatibilizer content. The incorporation of agave fiber reduced the crystallinity of the composites. High agave fiber content (25 wt \%) showed a decrease of 10 and $15 \%$ in $X_{c}$ for PP and rLLDPE components in the composites, respectively, because of the reduction in the amount of polymer and fibers 

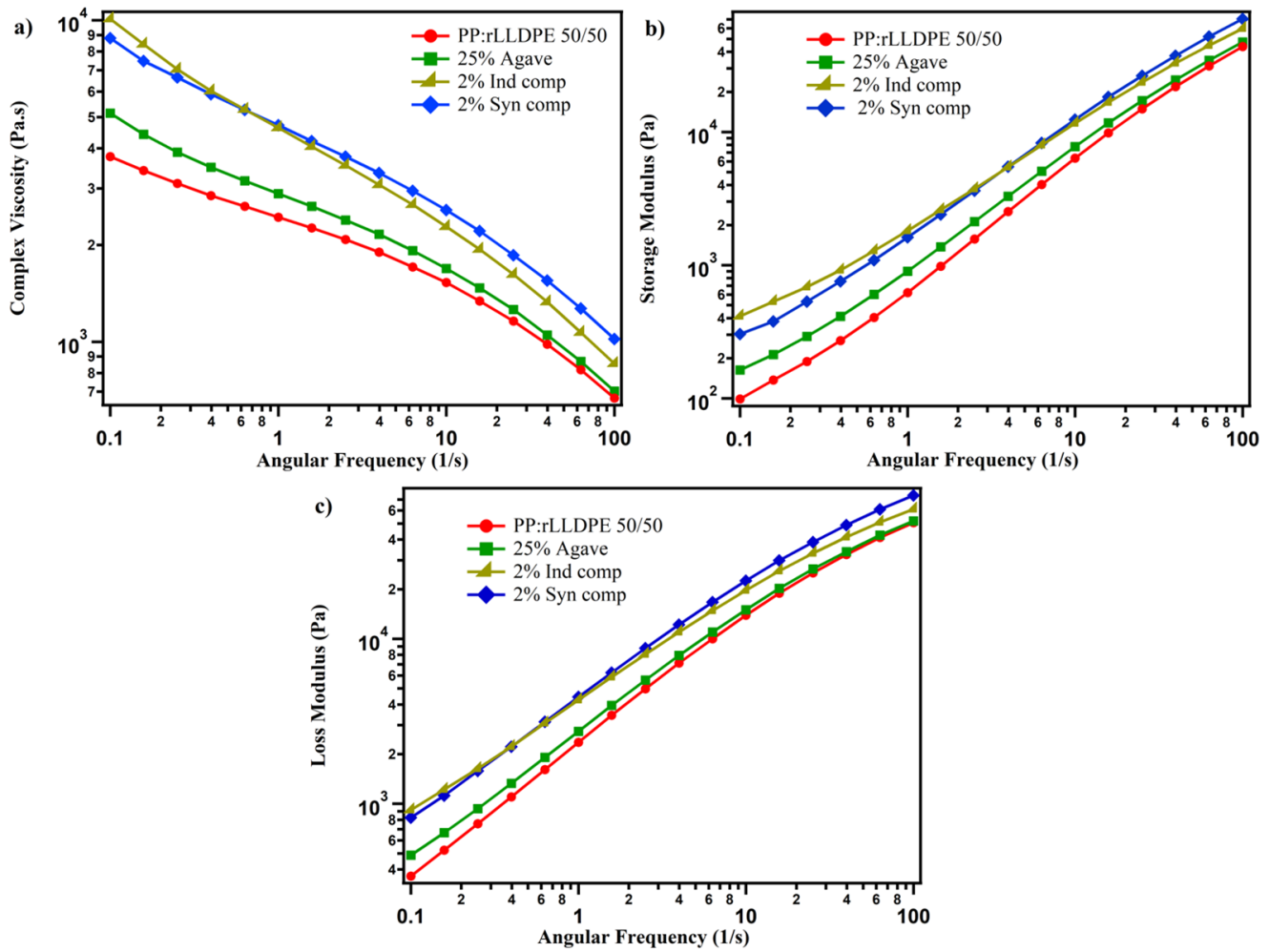

Figure 5. Rheological properties (a) complex viscosity, (b) storage modulus $\mathrm{G}^{\prime}$, and (c) loss modulus $\mathrm{G}^{\prime \prime}$ of the PP/rLLDPE 50:50 matrix, 25\% agave; $2 \%$ industrial composites (ind comp), and $2 \%$ synthesized composites (syn comp) as a function of angular frequency at $180{ }^{\circ} \mathrm{C}$ and fixed strain of $1 \%$.

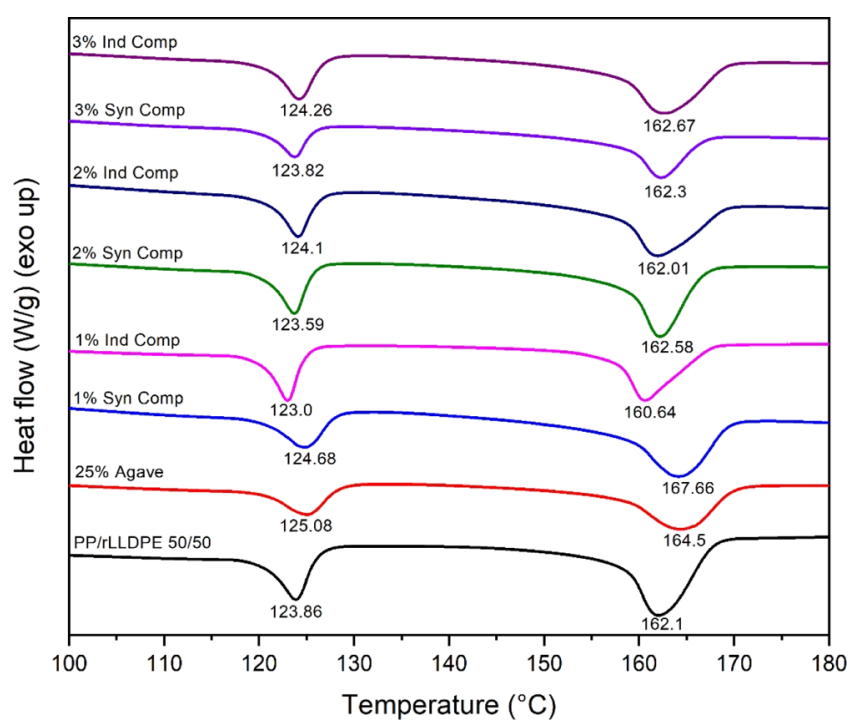

Figure 6. DSC second heating curve of the composites.

restricting the movement of the polymer chains, ${ }^{32,36}$ which is also supported by the increased tensile and flexural moduli. However, the composite with 2 wt \% compatibilizer showed higher PP crystallinity than that of the uncompatibilized samples, which is also supported by the fact that it had better mechanical properties than the others.

2.7. Density Characteristics. Density plays a significant role in determining the final weight of the product. Lowdensity composites are an excellent choice for automotive parts because of their role in decreasing the weight of the vehicles, which improves fuel efficiency. ${ }^{8}$ The key benefit of agave fiber as a filler material is that it has a much lower density $(1.2 \mathrm{~g} /$ $\mathrm{cm}^{3}$, according to our result) than commercial fibers, that is, glass fiber having a density of $2.6 \mathrm{~g} / \mathrm{cm}^{3} .{ }^{37}$ Density data of PP/ rLLDPE and its composites are presented in Table 3. The PP/ rLLDPE matrix has a density of $0.93 \mathrm{~g} / \mathrm{cm}^{3}$, which is increased by the addition of agave fiber. However, the addition of the compatibilizer did not show any change in the density with respect to the agave/matrix. The calculated densities of the formulations with the compatibilizer lie between 1.02 and 1.03 $\mathrm{g} / \mathrm{cm}^{3}$.

\section{CONCLUSIONS}

Sustainable composites were produced by injection-molding from a recycled bale wrap LLDPE/polypropylene (PP/ rLLDPE 50:50 by weight) matrix and 25 wt \% waste agave fiber with a compatibilizer content variation (1-3 wt \%). Incorporation of a laboratory-synthesized compatibilizer (MAgrafted PP/LLDPE blend) in the composite comparatively improved the interfacial adhesion between the polymers blends (PP and recycled LLDPE) and agave fiber more than the chosen industrial compatibilizer counterpart as studied in this investigation. Furthermore, the addition of the laboratorysynthesized compatibilizer increased the tensile and flexural properties of the composite without significant change in the impact strength as compared to uncompatibilized composites. The optimum sustainable composite was achieved using $2 \mathrm{wt}$ $\%$ synthesized compatibilizer with the tensile and flexural strength increasing by 24.4 and $14.1 \%$, respectively, with respect to the uncompatibilized composites. Moreover, the tensile and flexural moduli increased by 13.1 and $3.5 \%$ compared to the composite without the compatibilizer. HDT of the agave fiber composite with 2 wt $\%$ synthesized 
Table 2. Thermal Properties of PP/rLLDPE Composites from the Second DSC Heating Scan

\begin{tabular}{|c|c|c|c|c|c|c|}
\hline sample & \multicolumn{2}{|r|}{$\begin{array}{l}T_{\mathrm{m}}\left({ }^{\circ} \mathrm{C}\right) \\
\text { second heating }\end{array}$} & \multicolumn{2}{|c|}{$\begin{array}{c}\Delta H_{\mathrm{m}}(\mathrm{J} / \mathrm{g}) \\
\text { second heating }\end{array}$} & \multicolumn{2}{|c|}{$\begin{array}{c}X_{c}(\%) \\
\text { second heating }\end{array}$} \\
\hline PP & \multicolumn{2}{|r|}{$163.5(0.3)$} & \multicolumn{2}{|c|}{$103.8(0.7)$} & \multicolumn{2}{|c|}{$50.1(0.5)$} \\
\hline rLLDPE & \multicolumn{2}{|r|}{$124.3(0.02)$} & \multicolumn{2}{|c|}{$74.8(7.74)$} & \multicolumn{2}{|c|}{$25.52(1.35)$} \\
\hline PP/rLLDPE & PP & rLLDPE & PP & rLLDPE & PP & rLLDPE \\
\hline$(50: 50)$ & $163.0(1.1)$ & $124.3(0.6)$ & $41.5(0.46)$ & $14.1(4.1)$ & $40.1(0.5)$ & $9.65(2.8)$ \\
\hline $25 \%$ agave & $164.7(0.2)$ & $125.1(0.1)$ & $30.9(1.1)$ & $11.7(2.1)$ & $39.8(1.4)$ & $10.1(1.9)$ \\
\hline $2 \%$ ind comp & $163.6(2.2)$ & $124.9(1.2)$ & $32.3(3.1)$ & $11.1(2.6)$ & $42.7(4.1)$ & $10.4(2.4)$ \\
\hline $2 \%$ syn comp & $164.4(3.0)$ & $125.0(1.7)$ & $31.6(3.1)$ & $10.7(2.1)$ & $41.9(4.2)$ & $10.7(2.1)$ \\
\hline
\end{tabular}

Table 3. Density of the PP/rLLDPE Matrix and the Composites

\begin{tabular}{ll}
\multicolumn{1}{c}{ Formulation } & Density $\left(\mathrm{g} / \mathrm{cm}^{3}\right)$ \\
$\mathrm{PP} / \mathrm{rLLDPE}$ matrix $(50: 50)$ & $0.929 \pm 0.0004$ \\
agave/matrix $(25 / 75)$ & $1.020 \pm 0.002$ \\
agave/matrix/synthesized compatibilizer $(25 / 73 / 2)$ & $1.030 \pm 0.005$ \\
agave/matrix/industrial compatibilizer $(25 / 73 / 2)$ & $1.016 \pm 0.0012$ \\
\hline
\end{tabular}

compatibilizer showed around $18 \%$ enhancement compared to the composite without a compatibilizer. SEM showed poor adhesion between the matrix and the agave fibers. However, addition of the compatibilizer helped in the adhesion and interaction between the matrix and agave fiber, as confirmed from FTIR analysis. Moreover, the adhesion between the matrix and agave was also confirmed by the increase in the complex viscosity and storage modulus of PP/rLLDPE/25 wt $\%$ agave fiber composites after the inclusion of the compatibilizer. This type of novel sustainable and recycled plastic-based composite could be widely used in manufacturing packaging materials and commodity products and decreased the waste produced from non-degradable polymers.

\section{MATERIALS AND METHODS}

4.1. Materials. The waste agave fiber was acquired from the city of Tequila, Mexico. All the details of the cleaning and sieving of the agave fiber $(425-500 \mu \mathrm{m})$ have been described elsewhere. ${ }^{15}$ After sieving the fiber, it was oven-dried at $75{ }^{\circ} \mathrm{C}$ prior to processing. An agriculture waste bale wrap produced from LLDPE was received from Don Nott, Nott Farms, Clinton, ON, Canada. The rLLDPE bale wrap was wiped and cleaned of compost and soil, dried in an oven at $80{ }^{\circ} \mathrm{C}$, and then extruded using a counter-rotating twin screw extruder, Leistritz (Germany) at $180{ }^{\circ} \mathrm{C}$ and screw speed of $100 \mathrm{rpm}$, followed by pelletizing. PP pellets from Pinnacle Polymers, USA, under the trade name PP $1350 \mathrm{~N}$ were mixed with rLLDPE (50:50 ratio) as the matrix. Two types of compatibilizers were used in the study to compare their effectiveness in the biocomposites. They were the industrial compatibilizer MAPP/MAPE (50:50 by weight) and synthesized compatibilizer MA(PP/rLLDPE). Industrial compatibilizers MAPP and MAPE under the trade name Fusabond P353 for MAPP and Fusabond M603 for MAPE were purchased from Dupont (NC, USA). The initiator Luperox 101 (2,5bis(tert-butyl-peroxy)-2,5-dimethyl-hexane) was purchased from Sigma-Aldrich, USA.

4.2. Preparation of the "Synthesized Compatibilizer" MA(PP/rLLDPE). The synthesized compatibilizer was prepared in the laboratory using grades of $\mathrm{PP}$ and recycled LLDPE. LLDPE was dried in an oven at $75{ }^{\circ} \mathrm{C}$ overnight before processing. The composition was at a weight ratio of
50:50 (PP/rLLDPE), with 2.5 phr of MA (Sigma-Aldrich, USA) and $0.5 \mathrm{phr}$ of initiator Luperox 101. The PP and rLLDPE pellets were mixed manually with MA powder in a plastic bag for $2 \mathrm{~min}$. The desired amount of the initiator was dispersed in $1 \mathrm{~g}$ of acetone to give uniform mixing of Luperox with the polymer pellets and left in a fume hood for $30 \mathrm{~min}$ before processing. $\mathrm{MA}(\mathrm{PP} / \mathrm{rLLDPE})$ was produced via reactive extrusion using a counter-rotating twin screw extruder, Leistritz (Germany) at $180{ }^{\circ} \mathrm{C}$ with a screw speed of $60 \mathrm{rpm}$. The compatibilizer strands were cooled in a water bath, pelletized, and kept for 3 days in a vacuum oven at $80{ }^{\circ} \mathrm{C}$ to remove the unreacted MA. The grafting degree was determined similarly to the procedure by Muthuraj et al. ${ }^{38}$ using the back-titration method. The percentage of grafting was found to be $3.43 \%$.

4.3. Biocomposite Fabrication. Waste LLDPE and PP pellets (50:50 ratio) were mixed before processing. The biocomposites with 25 wt \% agave fiber were fabricated by adding different amounts $(1,2,3 \%)$ of a synthesized or industrial compatibilizer (Table 1). The biocomposites were prepared at $180{ }^{\circ} \mathrm{C}$ by a twin-screw counter-rotating extruder with a screw speed of $100 \mathrm{rpm}$ and 2 min mixing followed by injection-molding (DSM, The Netherlands).

4.4. Property Measurement. The surface morphology of the prepared biocomposites was observed by SEM using a Phenom ProX (The Netherlands) at an accelerating voltage of $15 \mathrm{kV}$. The impact cryofractured biocomposites were goldcoated for $10 \mathrm{~s}$ before the examination.

Mechanical properties were tested in accordance with ASTM standard methods-ASTM D638 for tensile tests, ASTM D790 for flexural and ASTM D256 for notched Izod impact strength. Tensile and flexural testing used an Instron 3382 while impact testing used a Zwick Roell-HP25 impact tester, Germany. The tensile and flexural properties were measured at crosshead speeds of 50 and $14 \mathrm{~mm} / \mathrm{min}$, respectively.

HDT was measured using a three-point cantilever on a dynamic mechanical analyzer Q800, TA Instruments, using a 2 ${ }^{\circ} \mathrm{C} / \mathrm{min}$ heating rate and $0.455 \mathrm{MPa}$ load in accordance with ASTM D648. The result was taken as the mean of two replicates.

FTIR spectra of the biocomposites were obtained to help predict the fiber-matrix interaction by observing bond formation using a Nicolet 6700 (Thermo Fisher, USA) with a $4 \mathrm{~cm}^{-1}$ resolution.

The rheological characteristics of the PP/rLLDPE matrix and agave fiber composite samples were obtained using an Anton Paar MCR-302 rheometer. The measurements were made at a strain of $1 \%$ (within the linear viscoelastic region) using a parallel plate geometry and a temperature of $180{ }^{\circ} \mathrm{C}$. The plates had a gap of $1 \mathrm{~mm}$ while the plate diameter was 25 
$\mathrm{mm}$. The angular frequency was varied between 0.05 and 600 $\mathrm{rad} / \mathrm{s}$.

Thermal properties were measured using a differential scanning calorimeter (Q200 TA Instruments, USA). Samples of about $6 \mathrm{mg}$ were subjected to a heating/cooling/heating cycle from -70 to $250{ }^{\circ} \mathrm{C}$ under inert gas $\left(\mathrm{N}_{2}\right)$ with a heating and cooling rate of $10^{\circ} \mathrm{C} / \mathrm{min}$. Two samples were tested for each material. The crystallinity $\left(X_{c}\right)$ of PP and rLLDPE was calculated according to eq 1

$$
X_{c}=\frac{\Delta H_{m}}{(\varnothing) \Delta H_{m}^{*}} \times 100
$$

where $\Delta H_{m}$ is the melting enthalpy of the matrix, $\phi$ is the weight fraction of PP or rLLDPE in respective samples, and $\Delta H_{m}{ }^{*}$ is the $100 \%$ melting enthalpy of perfectly crystalline PP $(207.1 \mathrm{~J} / \mathrm{g})$ or $\operatorname{rLLDPE}(293 \mathrm{~J} / \mathrm{g})$ according to theoretical measurements.

Density measurement of the matrix and composite materials was made using an MD-300S densimeter, Alfa Mirage, Japan.

\section{ASSOCIATED CONTENT}

\section{SI Supporting Information}

The Supporting Information is available free of charge at https://pubs.acs.org/doi/10.1021/acsomega.0c05186.

Stress-strain curve of PP/rLLDPE (50:50) and biocomposites (PDF)

\section{AUTHOR INFORMATION}

\section{Corresponding Author}

Manjusri Misra - School of Engineering and Bioproducts Discovery and Development Centre, Department of Plant Agriculture, University of Guelph, Guelph, Ontario N1G 2W1, Canada; (1) orcid.org/0000-0003-2179-7699; Email: mmisra@uoguelph.ca

\section{Authors}

Iftekhar H. Chowdhury - School of Engineering and Bioproducts Discovery and Development Centre, Department of Plant Agriculture, University of Guelph, Guelph, Ontario N1G 2W1, Canada

Mohamed A. Abdelwahab - Bioproducts Discovery and Development Centre, Department of Plant Agriculture, University of Guelph, Guelph, Ontario N1G 2W1, Canada

Amar K. Mohanty - Bioproducts Discovery and Development Centre, Department of Plant Agriculture and School of Engineering, University of Guelph, Guelph, Ontario N1G 2W1, Canada; (c) orcid.org/0000-0002-1079-2481

Complete contact information is available at:

https://pubs.acs.org/10.1021/acsomega.0c05186

\section{Notes}

The authors declare no competing financial interest.

${ }^{\S}$ On leave from Department of Chemistry, Faculty of Science, Tanta University, Tanta, 31527, Egypt.

\section{ACKNOWLEDGMENTS}

The authors are grateful for financial support to carry out this research: i) The Agriculture and Agri-Food Canada (AAFC) and Competitive Green Technologies, Canada through the AgSci Cluster Program (project no. 054712); (ii) the Ontario Ministry of Agriculture, Food and Rural Affairs (OMAFRA)/ University of Guelph-Bioeconomy for the Industrial Uses
Research Program (project \# no. 030177); (iii) the Ontario Research Fund - Research Excellence (ORF RE9) project from the Ontario Ministry of Economic Development, Job Creation and Trade (project nos. 053970 and 054345); and (iv) the Natural Sciences and Engineering Research Council of Canada (NSERC), Canada Research Chair (CRC) program (project no. 460788). The authors would also like to thank Don Nott, Nott Farms, Clinton, ON, Canada, for the donation of recycled and cleaned bale wrap samples for this research.

\section{REFERENCES}

(1) Schnurr, R. E. J.; Alboiu, V.; Chaudhary, M.; Corbett, R. A.; Quanz, M. E.; Sankar, K.; Srain, H. S.; Thavarajah, V.; Xanthos, D.; Walker, T. R. Reducing marine pollution from single-use plastics (SUPs): A review. Mar. Pollut. Bull. 2018, 137, 157-171.

(2) Geyer, R.; Jambeck, J. R.; Law, K. L. Production, use, and fate of all plastics ever made. Science Advances 2017, 3, No. e1700782.

(3) Leyva-Porras, C.; Esneider-Alcalá, M. A.; Toxqui-Terán, A.; Márquez-Lucero, A.; Aguilar-Martínez, J. A. Effect of Molding Parameters on Young's Modulus of an Injection Molded Low-Density Polyethylene (LDPE). Ind. Eng. Chem. Res. 2013, 52, 5666-5671.

(4) Friedrich, K. Polymer composites for tribological applications. Advanced Industrial and Engineering Polymer Research 2018, 1, 3-39.

(5) Ahn, Y.; Jeon, J. H.; Park, J.-H.; Thenepalli, T.; Ahn, J. W.; Han, C. Effects of modified LDPE on physico-mechanical properties of HDPE/CaCO3 composites. Korean J. Chem. Eng. 2016, 33, 32583266.

(6) Singh, N.; Hui, D.; Singh, R.; Ahuja, I. P. S.; Feo, L.; Fraternali, F. Recycling of plastic solid waste: A state of art review and future applications. Composites, Part B 2017, 115, 409-422.

(7) Brackenridge, S. Ontario innovation will help reduce on-farm plastic waste. (accessed December 2020).

(8) Watt, E.; Abdelwahab, M. A.; Snowdon, M. R.; Mohanty, A. K.; Khalil, H.; Misra, M. Hybrid biocomposites from polypropylene, sustainable biocarbon and graphene nanoplatelets. Sci. Rep. 2020, 10, 10714.

(9) Nonato, R. C.; Bonse, B. C. A study of PP/PET composites: Factorial design, mechanical and thermal properties. Polym. Test. 2016, 56, 167-173.

(10) Rahimi, A.; García, J. M. Chemical recycling of waste plastics for new materials production. Nat. Rev. Chem. 2017, 1, 0046.

(11) Graziano, A.; Jaffer, S.; Sain, M. Review on modification strategies of polyethylene/polypropylene immiscible thermoplastic polymer blends for enhancing their mechanical behavior. J. Elastomers Plast. 2019, 51, 291-336.

(12) Mohanty, A. K.; Misra, M.; Hinrichsen, G. Biofibres, biodegradable polymers and biocomposites: An overview. Macromol. Mater. Eng. 2000, 276-277, 1-24.

(13) Singha, A. S.; Rana, R. K. Natural fiber reinforced polystyrene composites: Effect of fiber loading, fiber dimensions and surface modification on mechanical properties. Mater. Des. 2012, 41, 289297.

(14) López-Bañuelos, R. H.; Moscoso, F. J.; Ortega-Gudiño, P.; Mendizabal, E.; Rodrigue, D.; González-Núñez, R. Rotational molding of polyethylene composites based on agave fibers. Polym. Eng. Sci. 2012, 52, 2489-2497.

(15) Smith, M. K. M.; Paleri, D. M.; Abdelwahab, M.; Mielewski, D. F.; Misra, M.; Mohanty, A. K. Sustainable composites from poly(3hydroxybutyrate) (PHB) bioplastic and agave natural fibre. Green Chem. 2020, 22, 3906-3916.

(16) Mylsamy, K.; Rajendran, I. Influence of alkali treatment and fibre length on mechanical properties of short Agave fibre reinforced epoxy composites. Mater. Des. 2011, 32, 4629-4640.

(17) Graebling, D.; Muller, R.; Palierne, J. F. Linear viscoelastic behavior of some incompatible polymer blends in the melt. Interpretation of data with a model of emulsion of viscoelastic liquids. Macromolecules 1993, 26, 320-329. 
(18) Annandarajah, C.; Langhorst, A.; Kiziltas, A.; Grewell, D.; Mielewski, D.; Montazami, R. Study of Agave Fiber-Reinforced Biocomposite Films. Materials 2019, 12, 3189.

(19) Youssef, A. M.; El-Gendy, A.; Kamel, S. Evaluation of corn husk fibers reinforced recycled low density polyethylene composites. Mater. Chem. Phys. 2015, 152, 26-33.

(20) Lei, Y.; Wu, Q.; Yao, F.; Xu, Y. Preparation and properties of recycled HDPE/natural fiber composites. Compos. Appl. Sci. Manuf. 2007, 38, 1664-1674.

(21) Mohanty, A. K.; Vivekanandhan, S.; Pin, J.-M.; Misra, M. Composites from renewable and sustainable resources: Challenges and innovations. Science 2018, 362, 536-542.

(22) Myers, G. E.; Chahyadi, I. S.; Gonzalez, C.; Coberly, C. A.; Ermer, D. S. Wood Flour and Polypropylene or High Density Polyethylene Composites: Influence of Maleated Polypropylene Concentration and Extrusion Temperature on Properties. Int. J. Polym. Mater. Polym. Biomater. 1991, 15, 171-186.

(23) Abdelwahab, M. A.; Misra, M.; Mohanty, A. K. Injection molded biocomposites from polypropylene and lignin: Effect of compatibilizers on interfacial adhesion and performance. Ind. Crops Prod. 2019, 132, 497-510.

(24) Zhang, Z.; Wang, C.; Mai, K. Reinforcement of recycled PET for mechanical properties of isotactic polypropylene. Advanced Industrial and Engineering Polymer Research 2019, 2, 69-76.

(25) Muthuraj, R.; Misra, M.; Mohanty, A. K. Biocomposite consisting of miscanthus fiber and biodegradable binary blend matrix: compatibilization and performance evaluation. RSC Adv. 2017, 7, $27538-27548$.

(26) Gao, H.; Xie, Y.; Ou, R.; Wang, Q. Grafting effects of polypropylene/polyethylene blends with maleic anhydride on the properties of the resulting wood-plastic composites. Compos. Appl. Sci. Manuf. 2012, 43, 150-157.

(27) Langhorst, A.; Paxton, W.; Bollin, S.; Frantz, D.; Burkholder, J.; Kiziltas, A.; Mielewski, D. Heat-treated blue agave fiber composites. Composites, Part B 2019, 165, 712-724.

(28) Langhorst, A. E.; Burkholder, J.; Long, J.; Thomas, R.; Kiziltas, A.; Mielewski, D. Blue-Agave Fiber-Reinforced Polypropylene Composites for Automotive Applications. Bioresources 2017, 13, 820-835.

(29) Toriz, G.; Denes, F.; Young, R. A. Lignin-polypropylene composites. Part 1: Composites from unmodified lignin and polypropylene. Polym. Compos. 2002, 23, 806-813.

(30) Landel, R. F.; Nielsen, L. E., Mechanical properties of polymers and composites; CRC press, 1993.

(31) Zhang, X. M.; Ajji, A. Oriented structure of PP/LLDPE multilayer and blends films. Polymer 2005, 46, 3385-3393.

(32) Cisneros-López, E. O.; Anzaldo, J.; Fuentes-Talavera, F. J.; González-Núñez, R.; Robledo-Ortíz, J. R.; Rodrigue, D. Effect of agave fiber surface treatment on the properties of polyethylene composites produced by dry-blending and compression molding. Polym. Compos. 2017, 38, 96-104.

(33) Abdelwahab, M. A.; Rodriguez-Uribe, A.; Misra, M.; Mohanty, A. K. Injection molded novel biocomposites from polypropylene and sustainable biocarbon. Molecules 2019, 24, 4026.

(34) Abdennadher, A.; Vincent, M.; Budtova, T. Rheological properties of molten flax- and Tencel ${ }^{\circledR}$-polypropylene composites: Influence of fiber morphology and concentration. J. Rheol. 2016, 60, 191-201.

(35) Nair, K. C. M.; Kumar, R. P.; Thomas, S.; Schit, S. C.; Ramamurthy, K. Rheological behavior of short sisal fiber-reinforced polystyrene composites. Compos. Appl. Sci. Manuf. 2000, 31, 12311240.

(36) Tang, C. Y.; Chen, D. Z.; Tsui, C. P.; Uskokovic, P. S.; Yu, P. H. F.; Leung, M. C. P. Nonisothermal melt-crystallization kinetics of hydroxyapatite-filled poly(3-hydroxybutyrate) composites. J. Appl. Polym. Sci. 2006, 102, 5388-5395.

(37) Nyambo, C.; Mohanty, A. K.; Misra, M. Polylactide-Based Renewable Green Composites from Agricultural Residues and Their Hybrids. Biomacromolecules 2010, 11, 1654-1660.
(38) Muthuraj, R.; Misra, M.; Mohanty, A. K. Injection Molded Sustainable Biocomposites From Poly(butylene succinate) Bioplastic and Perennial Grass. ACS Sustainable Chem. Eng. 2015, 3, 27672776. 